
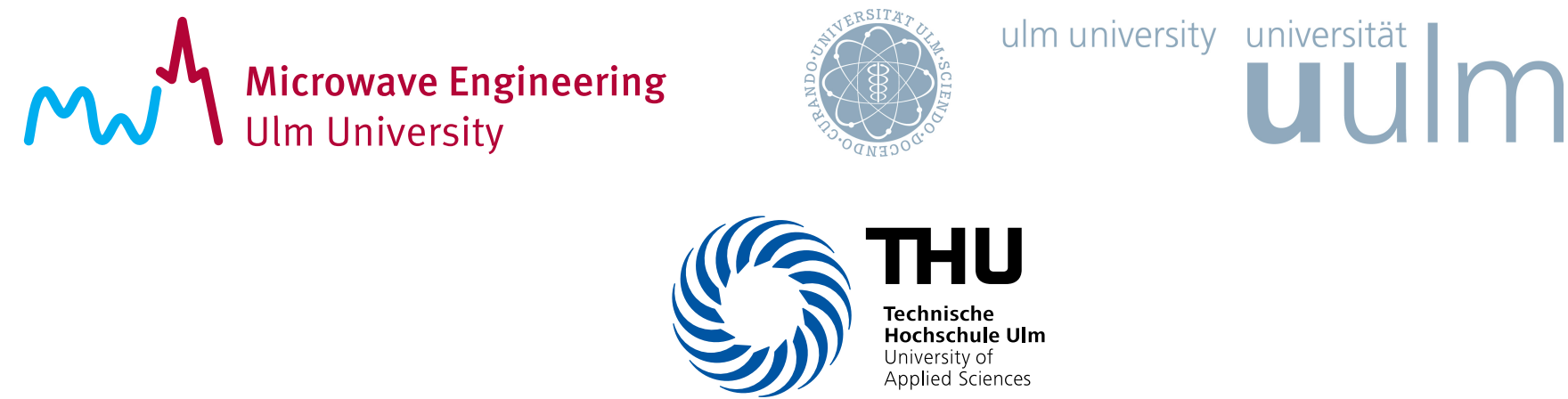

\title{
UAV-Based Polarimetric Synthetic Aperture Radar for Mine Detection
}

Ralf Burr, Markus Schartel, Winfried Mayer, Thomas Walter, and Christian Waldschmidt

(C) 2019 IEEE. Personal use of this material is permitted. Permission from IEEE must be obtained for all other uses, in any current or future media, including reprinting/republishing this material for advertising or promotional purposes, creating new collective works, for resale or redistribution to servers or lists, or reuse of any copyrighted component of this work in other works. 


\title{
UAV-BASED POLARIMETRIC SYNTHETIC APERTURE RADAR FOR MINE DETECTION
}

\author{
Ralf Burr $^{*}$, Markus Schartel ${ }^{\dagger}$, Winfried Mayer ${ }^{\ddagger}$, Thomas Walter ${ }^{*}$, and Christian Waldschmidt ${ }^{\dagger}$ \\ *Laboratory of Microtechnology, Ulm University of Applied Sciences, 89075 Ulm, Germany \\ $\dagger$ Institute of Microwave Engineering, Ulm University, 89081 Ulm, Germany \\ ${ }^{\ddagger}$ Endress+Hauser SE+Co. KG, 79689 Maulburg, Germany \\ Email: burr@hs-ulm.de
}

\begin{abstract}
In this contribution a polarimetric side-looking synthetic aperture radar (SAR) mounted on a unmanned aerial vehicle (UAV) is presented and discussed with respect to the detection and localization of landmines. As an example for an anti-personal mine a PFM-1 which contains an elongated aluminium rod was considered. Such anisotropic geometries exibit a polarization dependend radar cross section (RCS). Through a special configuration of three antennas, polarimetric SAR measurements involving a back projection algorithm could be implemented. This concept allows for the detection and furthermore the classification of such anisotropic objects. First field tests using a tachymeter for localization of the UAV over a snow covered meadow successfully demonstrated the performance by the detection of small metal rods depending on their orientation with respect to the flight path of the UAV. These experimental results were supported by simulations expressing the necessity of polarimetric measurements in combination with a distinct flight trajectory for a robust detection of certain landmines.
\end{abstract}

Index Terms - Anti-personnel mine, frequency-modulated continuous-wave radar, polarimetric synthetic aperture radar, unmanned aerial vehicle.

\section{INTRODUCTION}

Over the last decades, the use of anti-personnel mines (APM) as weapons in armed conflicts all over the world has drastically increased. Such weapons possess a fusing system which is sensitive to pressure leading to an explosion by if a person steps onto the mine. These weapons intend to injure or kill people, to disrupt the supply of resources during a military conflict and to hinder enemy movement in times of armed conflicts [1].

For large-area spreading, mines have been developed which are scattered from aircrafts and randomly distributed onto the ground. The PFM-1 (Fig. 1) is a type of mine known

\footnotetext{
The authors thank the Urs Endress foundation for supporting this work. https://www.ue-stiftung.org
}

as "butterfly mine" [2] which has been used extensively in Afghanistan. This mine exhibits an elongated aluminium cylinder in the center surrounded by an explosive filled plastic cover in the form of a butterfly wing. After the end of military conflicts, these explosive remnants of war (ERW) remain on the ground and pose a constant threat to the civilian population [3].

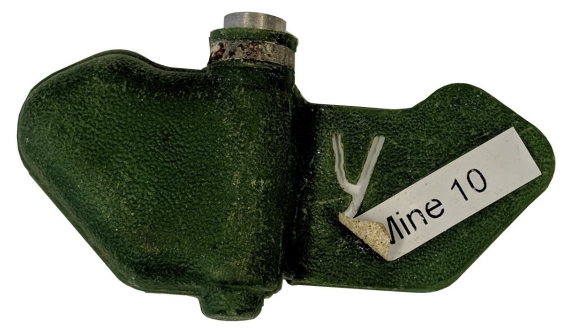

Fig. 1. PFM-1 cluster mine with a metallic cylindrical extended geometry in the center.

The primarily used equipment for mine detection are hand-held metal detectors (MD). As major disadvantage MDs have to be operated very close to the surface in a potentially hazardous area. A ground penetrating radar (GPR) represents a complementary sensor technology to the MD which can work from a greater distance and detect minimum metal nearsurface targets. In combination with an autonomous flying platform, a radar can be conducted over the impacted area without the need for human beings to enter this dangerous area.

Surfaces and interfaces result in a reflections and backscattering due to a gradient of the dielectric constant. This imposes a drawback of the radar-based UAV-supported mine detection as clutter from the ground complicates the detection of ERWs. In order to classify ERWs, the polarimetric properties of such objects can be used [4].

This paper is divided into four sections. After an introduction, section 2 introduces the system architecture of the radar equipped UAV. Section 3 discusses the polarimetric back-scattering properties of elongated aluminium rods in the frequency range $1 \mathrm{GHz}$ to $4 \mathrm{GHz}$. In section $4 \mathrm{SAR}$ measure- 
ments over a test field with different targets on the surface are presented.

\section{SYSTEM ARCHITECTURE}

A photograph of our system is displayed in Fig 2. Our system is based on a commercially available multicopter carrying a maximum payload of $6 \mathrm{~kg}$ with a flight time of up to $30 \mathrm{~min}$. The side-looking radar consists of one transmitting and two receiving antennas. A 3D-printed support structure with a weight of $210 \mathrm{~g}$ is the base of the linearly polarized TEM-horn antenna with an useable bandwidth from $0.6 \mathrm{GHz}$ to $8.0 \mathrm{GHz}[5]$.

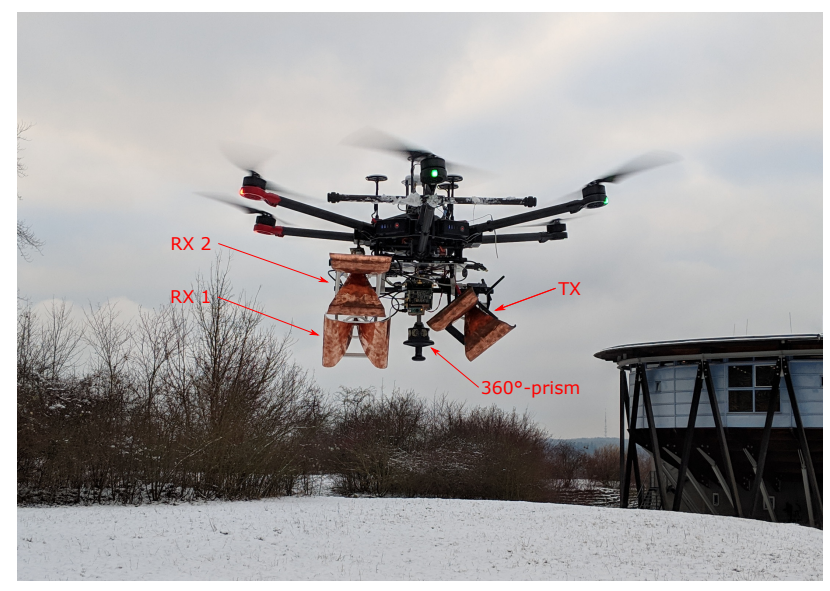

Fig. 2. Carrier platform with one transmitting antennas and two receiving antennas.

The GPR sensor itself consists of a main module and a receiver module. The main module contains the FMCW transmitter and the local oscillator for the receiver. The transmitter operates in the frequency range of $1 \mathrm{GHz}$ to $4 \mathrm{GHz}$ [6].

The configuration of the transmitting and receiving antennas enables the conduction of polarimetric SAR measurements.

For SAR processing, the exact trajectory of the flight path is necessary. Therfore a tachymeter is used tracking the $360^{\circ}$ prism attached to the UAV. The orientation of the UAV is deduced from the onboard inertial measurement unit (IMU) of the UAV. Data are stored on an onboard computer and processed after the flight.

\section{SIMULATIONS}

For a better understanding of the interaction between metallic cylindrical elongated objects and microwaves, 3D electromagnetic simulations have been undertaken. As a simple approach to the PFM-1, this mine is modelled as a metal rod with a diameter of $8 \mathrm{~mm}$ and a length of $60 \mathrm{~mm}$. Two configurations are simulated which are relevant for our measuring system presented in Sec. 2. These two configurations consist of metallic rods located on the surface parallel and orthogonal to the flight path. These two rod arrangements are stimulated with a plane wave source in horizontal and vertical polarization in the frequency range $1 \mathrm{GHz}$ to $4 \mathrm{GHz}$. In the first simulation, the directly backscattered RCS over frequency of the two configurations is calculated for vertical and horizontal polarization. The results are ilustrated in Fig. 3.

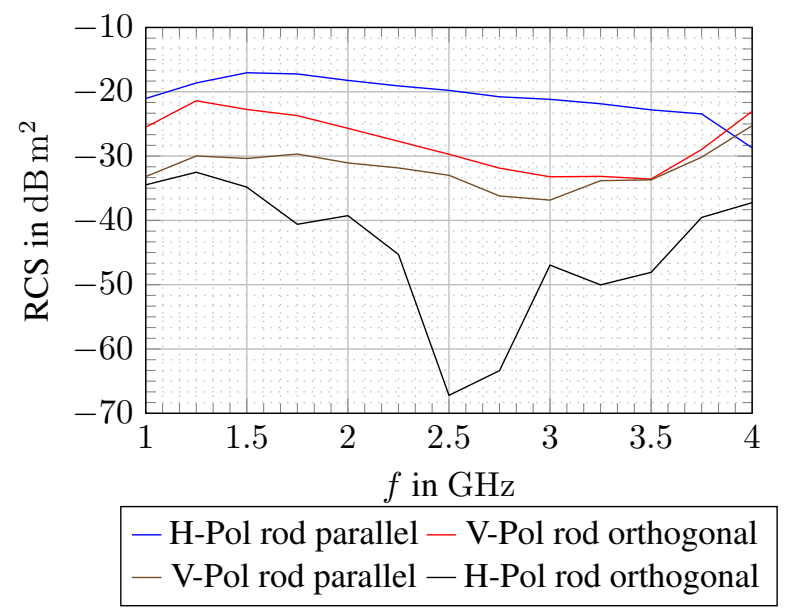

Fig. 3. Comparison of the RCS in the frequency range $1 \mathrm{GHz}$ to $4 \mathrm{GHz}$ for different polarizations as well as parallel and orthogonal alignment of the rod to the flight path.

With $-16 \mathrm{dBsm}$ at $2 \mathrm{GHz}$ the rod parallel to the flight path and horizontal polarization exibits the largest RCS. The orthogonal rod shows a maximum at vertical polarization with $-22 \mathrm{dBsm}$ for a frequency of $1.25 \mathrm{GHz}$. However, the RCS of the other two combinations is significantly lower especially in the frequency range of $1 \mathrm{GHz}$ to $3.5 \mathrm{GHz}$.

In order to evaluate qualitatively and discuss the results of the four configurations, the far fields were simulated at $3 \mathrm{GHz}$. The direction of incidence for the plane wave was set to $\theta=45^{\circ}$ and $\phi=0^{\circ}$. Fig. 4 illustrates the farfield of the parallel rod and horizontal polarization, whereas in Fig. 5 the farfield of the orthogonal rod and vertical polarization is shown.

In Fig. 4 and Fig. 5 a dipole like back-scattering pattern can be observed. The main beam direction of the orthogonal rod with vertical polarization does not point in the direction of the field source resulting in a lower RCS. In contrast, for the rod parallel to the flight path and horizontal polarization, the main beam direction points directly in the direction of the field source.

Fig. 6 illustrates the far field of the orthogonal rod with horizontal polarization and Fig. 7 displays the far field of the rod parallel to the flight path with vertical polarization.

From Fig. 6 and Fig. 7 it can be deduced that the rods seem to scatter more or less randomly. From Fig. 6 it is quite obvious that the rod mainly radiates primarily perpendicular 


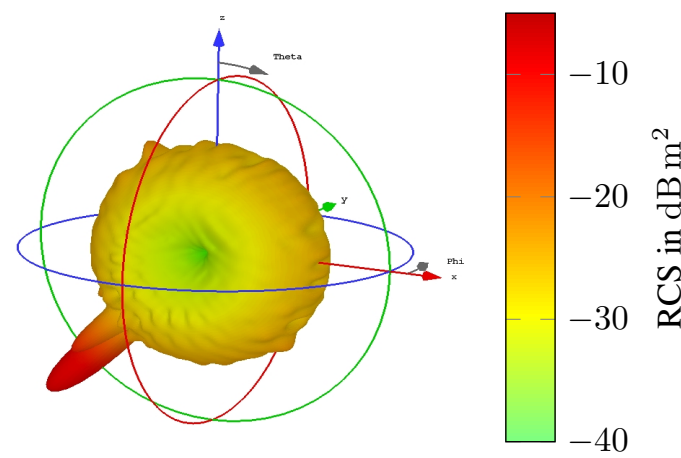

Fig. 4. RCS of rod parallel to trajectory and horizontal polarization at $3 \mathrm{GHz}$.
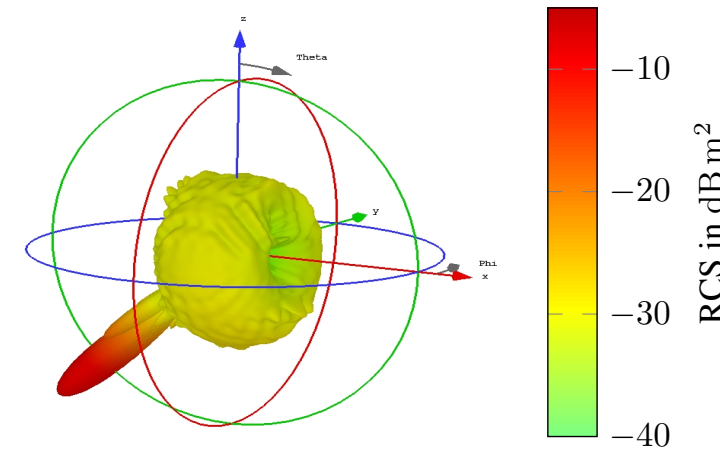

Fig. 5. RCS of rod orthogonal to trajectory and vertikal polarization at $3 \mathrm{GHz}$.

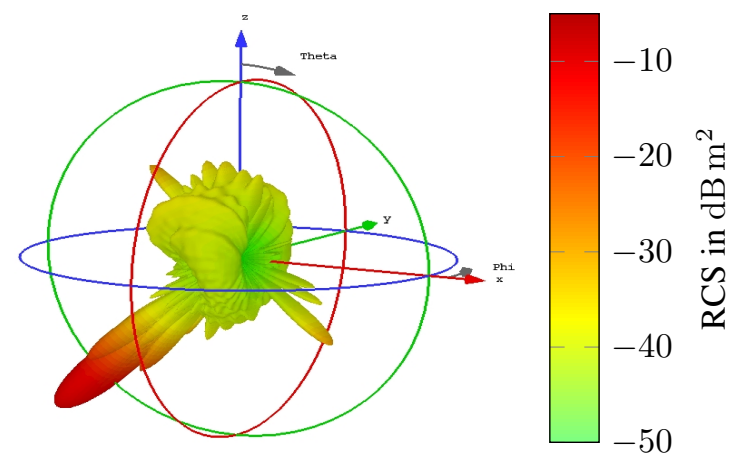

Fig. 6. RCS of rod orthogonal to trajectory and horizontal polarization at $3 \mathrm{GHz}$.

to the direction of the impinging plane wave explaining also the low RCS in Fig. 3. The scattering behaviour in Fig. 6 and Fig. 7 results from the ratio of the diameter of the rod and the wavelength. This can be explained by a transition from Rayleigh to Mie scattering. The simulations displayed above clearly demonstrate that the back-scattering or back-reflection and the associated RCS of the considered objects strongly depend on the polarization and object orientation with respect to the flight path.

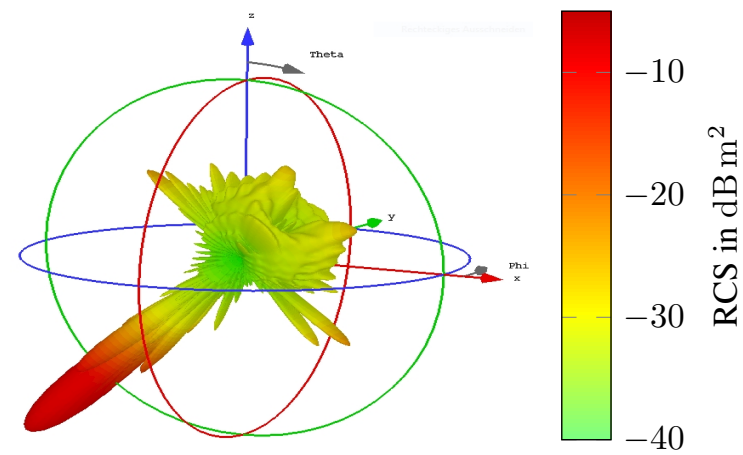

Fig. 7. RCS of rod parallel to trajectory and vertical polarization at $3 \mathrm{GHz}$.

\section{RESULTS}

\subsection{MEASUREMENT SETUP}

For the first tests two pairs of aluminium rods with a diameter of $8 \mathrm{~mm}$ and a length of $60 \mathrm{~mm}$ were placed parallel and perpendicular to the flight path on the ground surface covered with snow. The rods were located within two square arrangements of four reflectors each with a RCS of $-16 \mathrm{dBsm}$ and of four reflectors with a RCS of $-5 \mathrm{dBsm}$. The test setup is displayed in Fig. 8. The flight path runs nearly parallel to the two large reflectors at a distance of $2 \mathrm{~m}$ at an altitude of $3 \mathrm{~m}$. The meadow is covered with about $4 \mathrm{~cm}$ of snow with the first centimeter in depth of the soil being frozen.

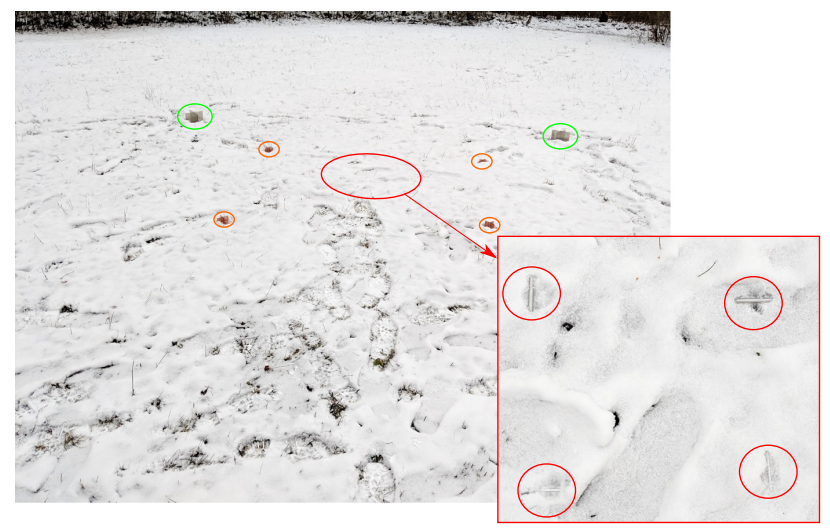

Fig. 8. Snow-covered field with two square arrangements of reflectors as well as aluminium rods in different orientations in the center.

\subsection{MEASUREMENT RESULTS}

The recorded time domain data for each channel are corrected by the offset of the phase centre in the time domain and subsequently transformed into the frequency domain with 16 times zero padding. The SAR image is focused with a backprojection algorithm presented in [7]. The SAR image for the 
channel with horizontal polarization is shown in Fig 9, for the vertical polarization in Fig. 10.

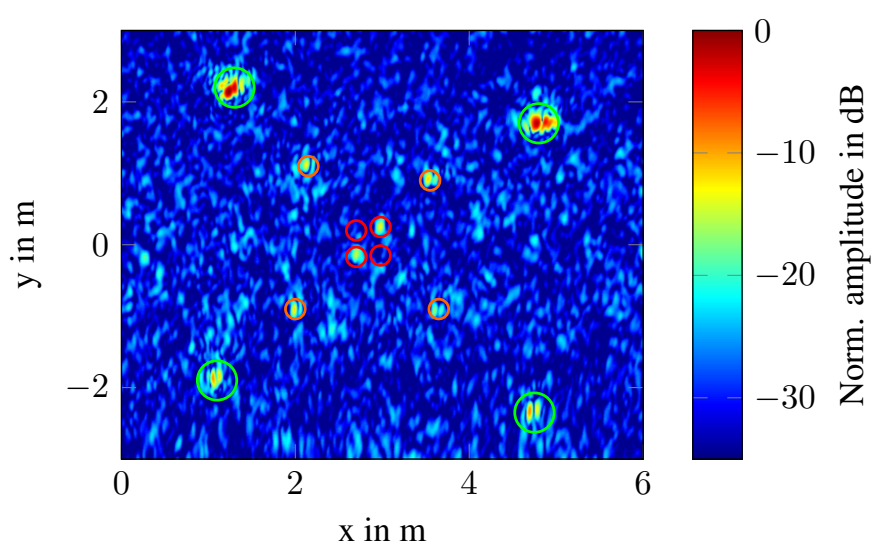

Fig. 9. SAR image of the in Fig. 8 shown setup with horizontally polarized receiving antenna.

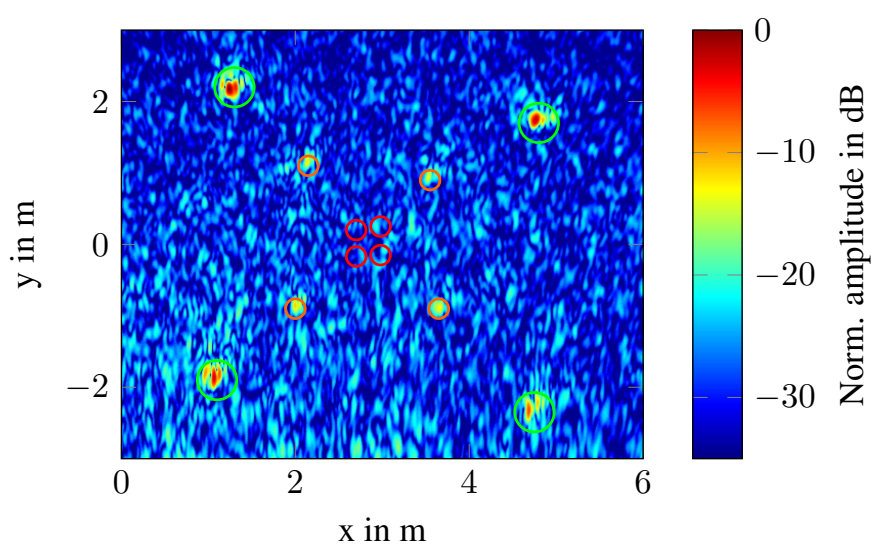

Fig. 10. SAR image of the in Fig. 8 shown setup with vertically polarized receiving antenna.

The positions of the two square arrangements consisting of large and small reflectors can be clearly observed in Fig. 9 and Fig. 10. However, in Fig. 9 only the rods parallel to the flight path can be detected. Thus, from these two polarimetric SAR images the orientation of the rods can be distinguished. In order to capture all four rods, an additional orthogonal flight trajectory is essential, which represents, however, no limitation to the UAV platform.

From the simulations in section 3 it can be deduced that the parallel rod can be detected by horizontal polarization, whereas the orthogonal rod exhibits a significantly lower RCS which complicates the robust detection in the presence of clutter being the case for our field test. It should be noted that for vertical polarization no robust detection at all is predicted.

\section{CONCLUSION}

In this contribution we demonstrated that based on a sidelooking polarimetric SAR mounted on an UAV cylindrical elongated metal rods could be detected and classified with respect to their orientation. A first flight over a snow covered meadow was successfully undertaken and objects similar to cluster mines could be detected. The polarization dependence of the detection can be explained by the orientation of the elongated metal objects with respect to the flight trajectory. This finding was supported by 3D EM simulations. The concept and results presented in this contribution demonstrate a promising method for an efficient mine detection.

\section{REFERENCES}

[1] Rasaq Bello, "Literature review on landmines and detection methods," Frontiers in Science, pp. 27-42, Vol. 3 No. 12013.

[2] Alex Nikulin, Timothy de Smet, Jasper Baur, William Frazer, and Jacob Abramowitz, "Detection and identification of remnant PFM-1 butterfly mines with a UAV-based thermal-imaging protocol," Remote Sensing, vol. 10, no. 11, pp. 1672, Oct 2018.

[3] The International Campaign to Ban Landmines, "Landmine monitor 2016," Monitoring and Research Committee, ICBL-CMC Governance Board, November 2016.

[4] X. Feng, E. Nilot, M. Zhang, and S. Liang, "Full polarimetric gpr data decomposition and imaging," in 2016 IEEE International Geoscience and Remote Sensing Symposium (IGARSS), July 2016, pp. 7477-7480.

[5] R. Burr, M. Schartel, W. Mayer, T. Walter, and C. Waldschmidt, "Lightweight broadband antennas for UAV based GPR sensors," in 48th European Microwave Conference (EuMC), September 2018, pp. 1-4.

[6] Ralf Burr, Markus Schartel, Patrick Schmidt, Winfried Mayer, Thomas Walter, and Christian Waldschmidt, "Design and implementation of a FMCW GPR for UAV-based mine detection," in IEEE MTT-S International Conference on Microwaves for Intelligent Mobility (ICMIM 2018).

[7] M. Schartel, R. Burr, W. Mayer, N. Docci, and C. Waldschmidt, "UAV-based ground penetrating synthetic aperture radar," in IEEE MTT-S International Conference on Microwaves for Intelligent Mobility (ICMIM), April 2018, pp. 1-4. 